Revue des patrimoines

$29 \mid 2016$

Ensembles mobiliers, industriels, techniques.

Connaissance, protection, conservation, présentation au public

\title{
La constitution du patrimoine scientifique $d u$ musée Curie
}

Building up the Curie museum's scientific heritage

Renaud Huynh et Adrien Klapisz

\section{CpenEdition}

\section{Journals}

Édition électronique

URL : http://journals.openedition.org/insitu/12979

DOI : 10.4000/insitu. 12979

ISSN : $1630-7305$

Éditeur

Ministère de la Culture

Référence électronique

Renaud Huynh et Adrien Klapisz, «La constitution du patrimoine scientifique du musée Curie », In Situ [En ligne], 29 | 2016, mis en ligne le 13 juillet 2016, consulté le 10 décembre 2020. URL : http:// journals.openedition.org/insitu/12979; DOI : https://doi.org/10.4000/insitu.12979

Ce document a été généré automatiquement le 10 décembre 2020.

\section{(c) (i) $(9)$}

In Situ Revues des patrimoines est mis à disposition selon les termes de la licence Creative Commons Attribution - Pas d'Utilisation Commerciale - Pas de Modification 4.0 International. 


\title{
La constitution du patrimoine scientifique du musée Curie
}

\author{
Building up the Curie museum's scientific heritage
}

Renaud Huynh et Adrien Klapisz

\section{Introduction}

1 Pour appréhender la constitution progressive du patrimoine scientifique du musée Curie, il est nécessaire d'examiner la succession d'événements qui ont conduit à la création d'un ensemble qui regroupe aujourd'hui des lieux, des objets, des archives et des documents au sein de l'ancien Institut du radium, devenu Institut Curie, un centre de recherche et de soins spécialisé dans la lutte contre le cancer.

2 Le musée Curie, labellisé «Maison des Illustres» en 2013, attire aujourd'hui des visiteurs du monde entier autour de ses collections et de la figure emblématique de Marie Curie (1867-1934). Le bureau qu'elle a occupé de 1914 à 1934, son laboratoire de chimie, de même que le petit jardin et les bâtiments de l'ancien Institut du radium, ainsi qu'une partie du matériel du laboratoire, ont été préservés et progressivement enrichis.

3 Quelles raisons ont conduit à la préservation de ce patrimoine? Quelle est la nature des collections et comment cet ensemble s'est-il constitué ?

4 Cet article propose une restitution chronologique des événements qui nous permettent de dégager des clés de compréhension du lent processus de " patrimonialisation » et de faire un état des lieux de la situation actuelle du patrimoine scientifique du musée Curie pour tenter d'en définir les contours et les spécificités.

\section{L'Institut du radium}

5 C'est en 1909 que l'Université de Paris et l'Institut Pasteur s'accordent pour créer un Institut du radium à Paris, à l'instar de ceux de Londres ou de Vienne. L'architecte de la 
Sorbonne, Henri-Paul Nénot, élabore les plans des laboratoires et des bâtiments dédiés aux recherches sur les rayonnements et aux études de leurs effets biologiques. La construction de l'Institut du radium s'étend sur plusieurs mois, de 1911 à 1914. L'un des bâtiments abrite le laboratoire Curie, placé sous la direction de Marie Curie, professeur titulaire de la chaire de physique générale et radioactivité à la faculté des sciences. C'est elle qui dirige le laboratoire dont Pierre Curie (1859-1906) avait rêvé et qu'il ne connaîtra jamais, victime d'un accident de circulation en 1906.

Le laboratoire Curie de l'Institut du radium (fig. 1) est le troisième et dernier laboratoire où Marie Curie a travaillé, dans des conditions plus confortables que celles qu'elle a partagées avec son époux, à l'École municipale de physique et de chimie industrielles de Paris. Ensemble, ils y découvrirent en 1898 le polonium et le radium, puis prolongent leurs travaux sur la radioactivité dans un petit laboratoire de la rue Cuvier, à partir de 1904. Pendant vingt ans, de l'installation de son laboratoire en 1914 jusqu'à son décès en 1934, Marie Curie met toute son énergie dans le développement des études sur les substances radioactives à l'Institut du radium. Elle fait de son laboratoire un des hauts lieux de la recherche scientifique en France et dans le monde.

Figure 1

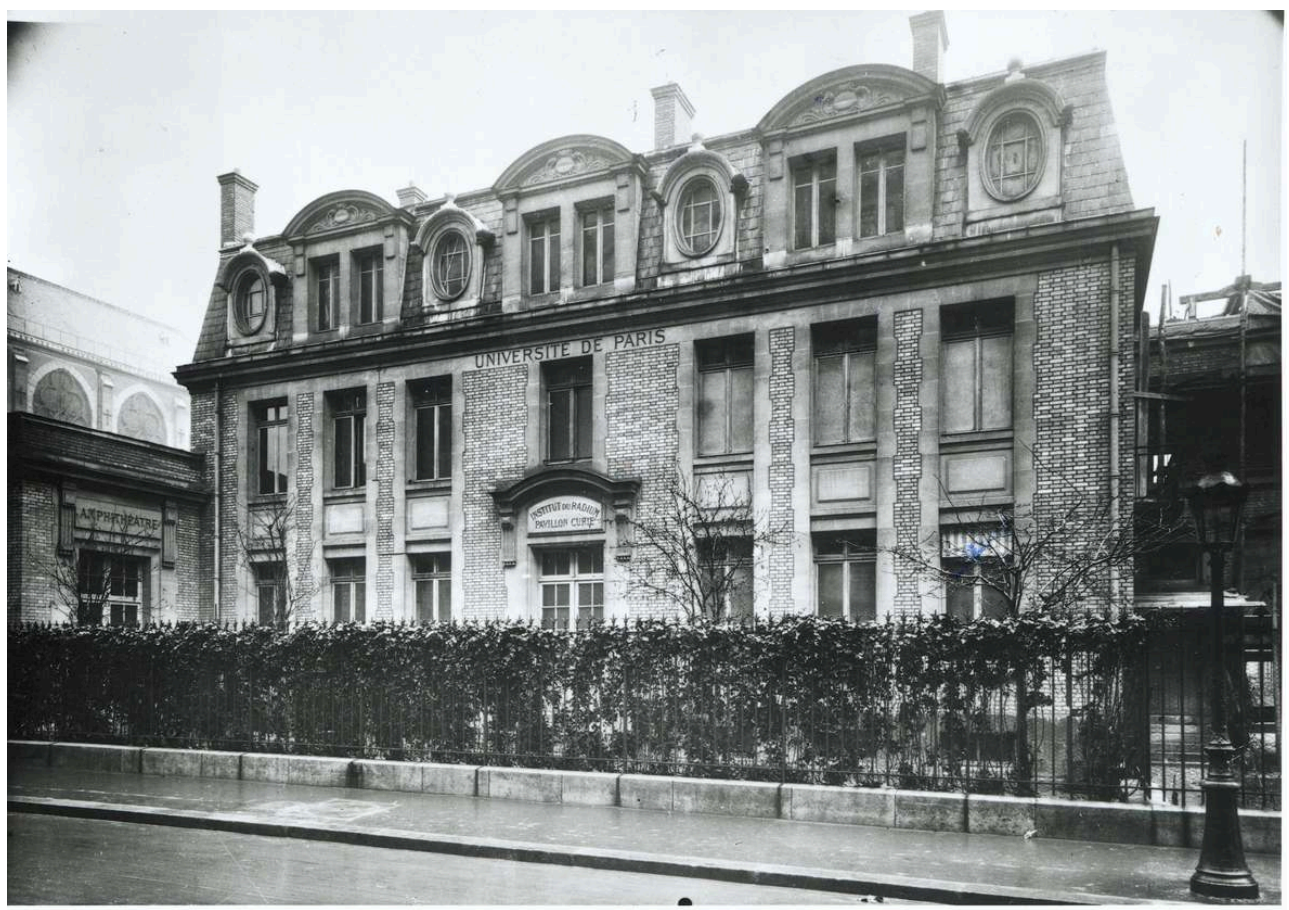

Façade du pavillon Curie de l'Institut du radium de Paris, vers 1920.

(C) Musée Curie/coll. ACJC.

7 Irène Curie (1897-1956), fille aînée du couple de savants, travaille à l'Institut du radium pendant plus de quarante ans, d'abord étudiante-assistante, puis préparatrice, et enfin professeur. En 1926, elle épouse Frédéric Joliot (1900-1958), jeune ingénieur rencontré deux ans plus tôt au laboratoire. Leurs recherches communes les conduisent à la découverte de la radioactivité artificielle, récompensée par le prix Nobel de chimie en 1935 (une plaque apposée en 2000 à l'entrée du bâtiment rappelle cet événement). Lorsque Marie Curie décède en 1934, c'est le directeur adjoint, André Debierne (1874-1949), chimiste découvreur de l'actinium en 1899 et proche collaborateur des 
Curie, qui lui succède à la direction du laboratoire Curie, jusqu'à son départ en retraite, où il est remplacé par Irène Curie. Pendant dix ans, de 1946 à 1956, elle utilise le bureau directorial que sa mère occupait trente années auparavant (fig. 2, fig. 3). En 1958, à la suite du décès de Frédéric Joliot-Curie, directeur du laboratoire pendant les deux dernières années de sa vie, le bureau, le laboratoire attenant et une petite pièce contenant des archives sont préservés.

\section{Figure 2}

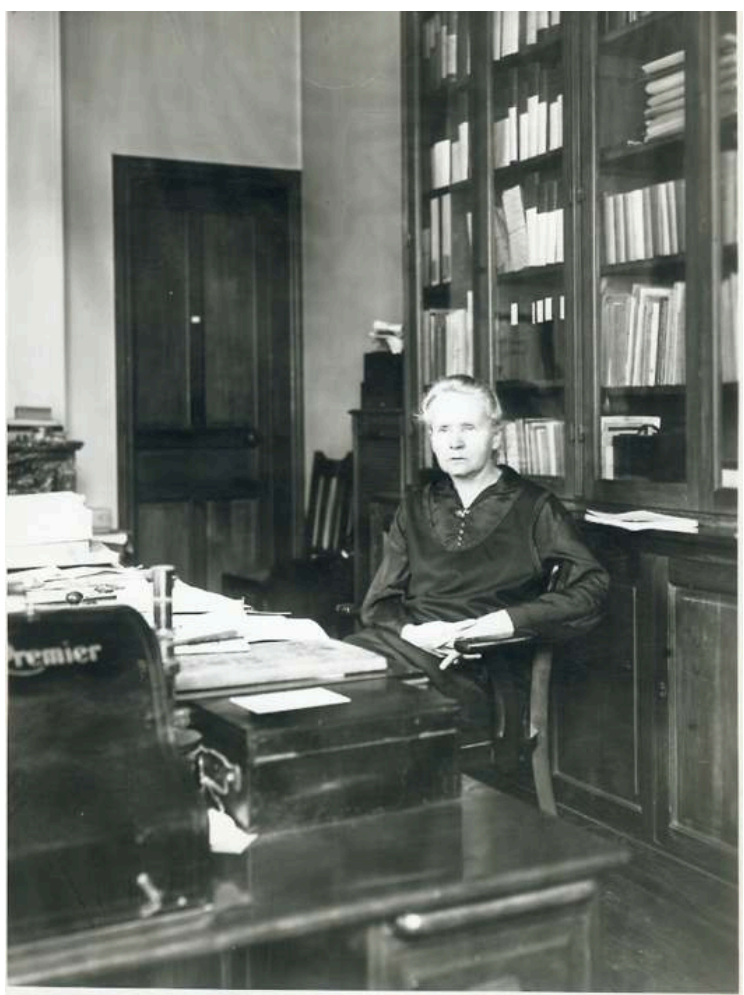

Marie Curie dans son bureau en 1928.

(c) Musée Curie/coll. ACJC. 


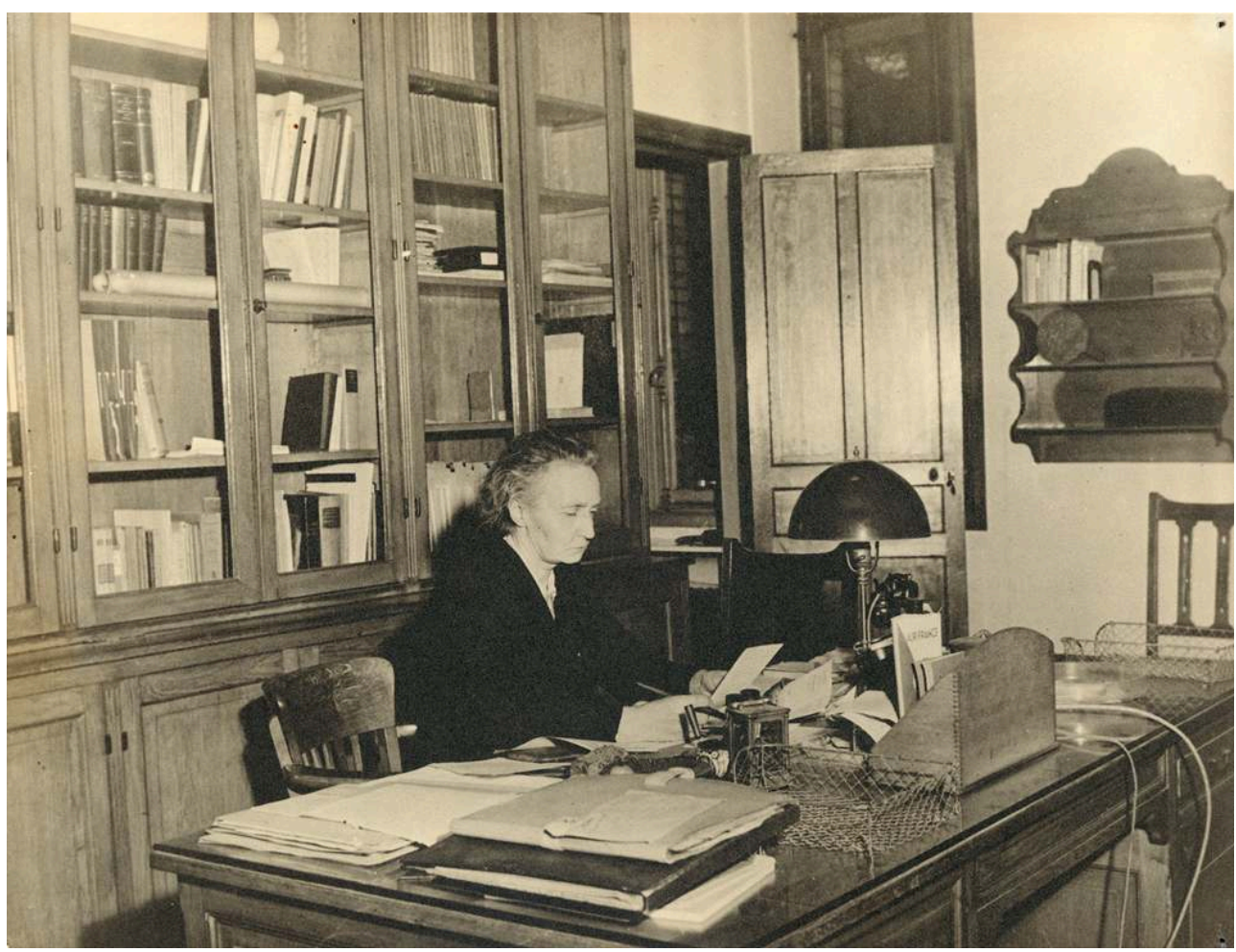

Irène Curie dans son bureau en 1956.

(c) Musée Curie/coll. ACJC.

8 À partir de cette date, ces lieux, inutilisés par les directeurs suivants, sont sauvegardés. Cette «sanctuarisation» de lieux de travail, qui perdent ainsi leur fonction initiale, constitue une première étape dans le processus de « patrimonialisation ».

\section{La constitution d'un patrimoine scientifique}

La transformation de locaux à vocation scientifique en lieux historiques est en effet l'étape initiale, le premier acte fondateur du musée Curie.

Les raisons de la conservation du bureau et du laboratoire, alloués successivement à Marie Curie, André Debierne, Irène puis Frédéric Joliot-Curie, sont multiples, mais la personnalité de Frédéric Joliot-Curie a sans doute participé à la préservation de ces pièces. En 1958, des funérailles nationales sont décrétées par le général de Gaulle à la mort de ce scientifique, prix Nobel et président du conseil mondial pour la Paix : le décès de Frédéric Joliot-Curie, comme celui de son épouse Irène Curie deux ans plus tôt, est un événement national. Le professeur Jean Teillac (1920-1994), qui succède à JoliotCurie à la direction du laboratoire de l'Institut du radium, décide de s'installer à proximité des nouveaux laboratoires de physique nucléaire à Orsay, renonçant ainsi à occuper le bureau laissé vacant et chargé de la mémoire de la première directrice, Marie Curie.

Par ailleurs, l'Association Irène et Frédéric Joliot-Curie (AJC) est créée en 1959, dans le but de préserver et perpétuer la mémoire du couple de savants récemment disparu. Elle regroupe les amis, les parents et les collaborateurs de Frédéric et Irène Joliot-Curie. Ses 
missions consistent à faire connaître la vie et l'œuvre des Joliot-Curie, leur engagement en faveur de la recherche scientifique, du progrès social et de la paix. C'est un acteur essentiel qui initie le regroupement d'objets mobiliers et la constitution d'un ensemble patrimonial.

Dès son origine, l'activité principale de l'association est d'organiser, de participer à des conférences, des commémorations, des expositions (par le prêt de documents et d'appareils scientifiques du laboratoire) ainsi qu'au rassemblement d'archives (documents, correspondances, photographies, etc.) qui conduisent à diverses publications.

Le 30 juin 1964, à l'occasion du congrès international de Physique nucléaire, organisé pour le $30^{\mathrm{e}}$ anniversaire de la découverte de la radioactivité artificielle, une exposition est inaugurée à l'entrée de l'Institut du radium (fig. 4). La concrétisation de ce projet est due principalement à la volonté d'anciens collaborateurs de Frédéric Joliot, et de sa fille, Hélène Langevin-Joliot, elle-même physicienne. Les $30 \mathrm{~m}^{2}$ de l'espace d'exposition, qui prennent place dans l'ancien laboratoire de physique de Marie Curie, proposent aux visiteurs de découvrir les travaux des Joliot-Curie à travers des archives et instruments scientifiques particulièrement significatifs, auxquels s'ajoutent quelques instruments conservés à leur domicile d'Antony et rapportés par leurs enfants pour l'occasion. À cette époque, la notoriété des Joliot-Curie éclipse provisoirement celle des Curie, notamment parce que les scientifiques impliqués dans la conception de l'exposition sont de proches collaborateurs de la deuxième génération de savants. La collection d'instruments scientifiques se construit donc principalement autour d'objets du laboratoire et d'appareils utilisés par les Joliot-Curie ou leurs contemporains. Une vitrine est cependant consacrée à Pierre et Marie Curie par le biais d'instruments de mesure de la radioactivité. 
Figure 4

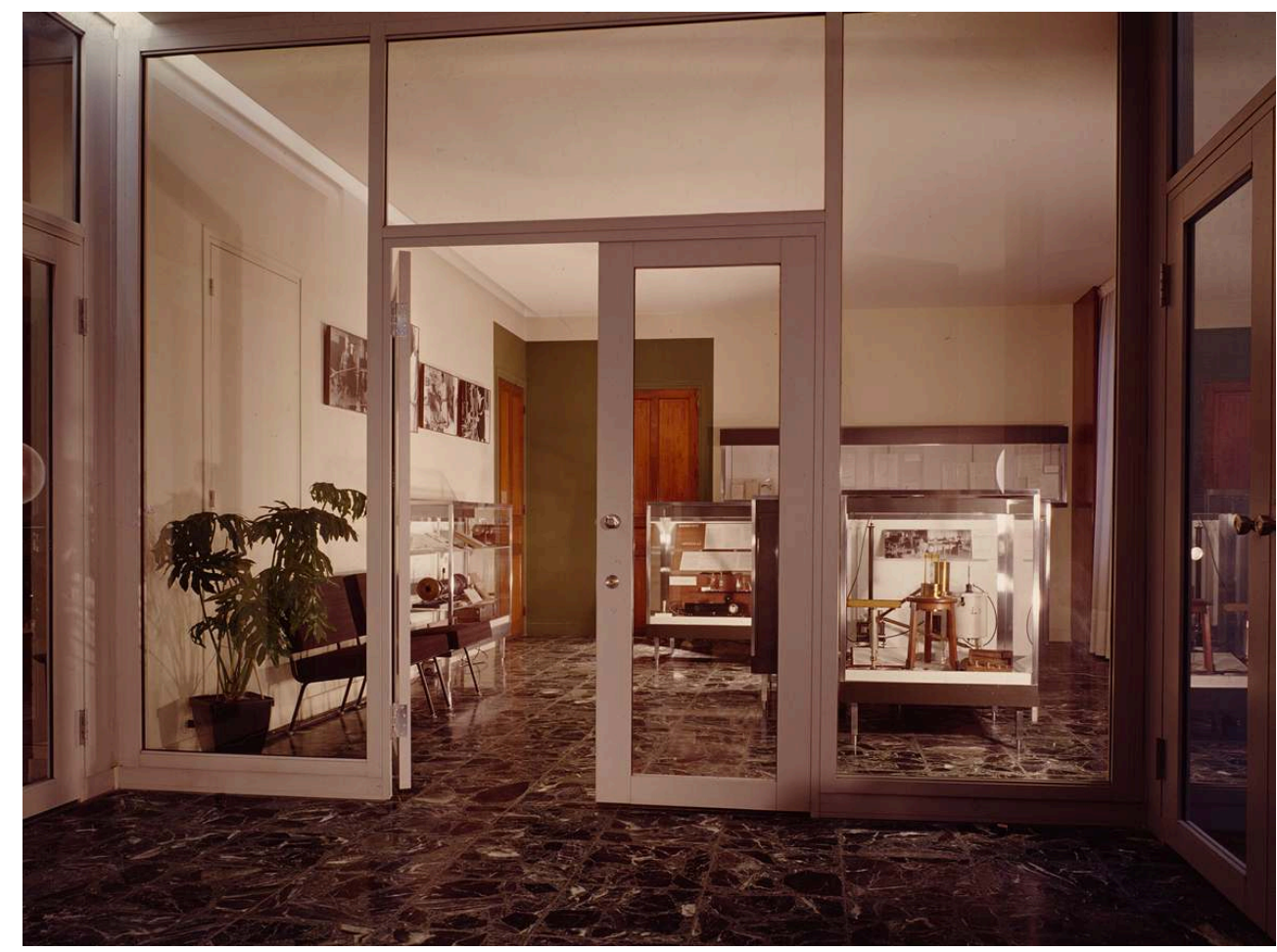

Salle d'exposition à l'entrée du pavillon Curie de l'Institut du radium, 1964.

(c) Musée Curie/coll. ACJC.

Cet espace de visite a été ainsi mis en scène à l'entrée du laboratoire dès 1964 par des scientifiques, pour la plupart physiciens nucléaires, avec l'intention de présenter au public les grandes étapes de l'histoire de la physique, à travers des vitrines qui présentent des instruments désormais obsolètes, statiques, même s'ils ont permis de grandes découvertes : ils trouvent ainsi une nouvelle fonction, non plus scientifique et pédagogique, mais historique et culturelle.

En 1967, dans le cadre des commémorations organisées pour le centenaire de la naissance de Marie Curie, les lieux sont présentés à des personnalités et scientifiques étrangers. La rue Pierre-Curie, créée en 1910 pour accéder à l'Institut du radium, est rebaptisée rue Pierre-et-Marie-Curie à cette occasion. Ève Curie-Labouisse, seconde fille de Pierre et Marie Curie, décide alors de séparer les archives de ses parents: une grande partie des documents personnels et des archives scientifiques des Curie sont donnés à la Bibliothèque nationale de France $(\mathrm{BnF})$, tandis que les autres documents, postérieurs à la construction du bâtiment en 1914, sont conservés in situ, dans l'ancien laboratoire de l'Institut du radium. Un agent est détaché de son poste à l'Institut de physique nucléaire d'Orsay, suite à une demande de l'AJC au CNRS, pour classer les archives et les documents du laboratoire Curie. D'abord seule, aidée ponctuellement par quelques membres de l'AJC, Monique Bordry identifie les documents, les étudie et les communique aux historiens et auteurs qui en font la demande, tout en organisant des visites guidées. Les activités de classement, d'inventaire et de diffusion des archives sont dès lors prédominantes.

Petit à petit, les fonds documentaires se structurent et les collections s'enrichissent à plusieurs reprises, principalement par des dépôts de la famille ou des dons d'anciens 
collaborateurs, de leurs descendants ou encore d'admirateurs et collectionneurs. Les efforts sont alors concentrés sur l'inventaire détaillé des archives du laboratoire Curie sous la direction de Marie Curie (1914-1934).

17 Suite à une demande de plus en plus insistante des publics, français et étrangers, l'ouverture d'un «musée du radium » est alors envisagée. La popularité de ce «lieu de mémoire » grandit, mais officiellement, il n'est pas ouvert au public : la radioactivité résiduelle, même faible, qui persistait dans certaines pièces doit être préalablement retirée et l'accueil des publics organisé. Le reste du bâtiment, au sous-sol, au rez-dechaussée et dans les étages, continue d'accueillir des activités scientifiques et des laboratoires de recherche, ce qui est toujours le cas aujourd'hui.

En 1981, le petit laboratoire de chimie qui jouxte le bureau utilisé par Marie Curie est décontaminé grâce au soutien de la Ligue française contre le cancer (fig. 5, fig. 6). L'ancienne paillasse de lave, contaminée par le radium, ainsi que les "taches" radioactives résiduelles, sont éliminées. Le laboratoire est ensuite reconstitué, sans toutefois accorder une grande importance à la réalité historique et à la mise en scène de cette pièce. La scénographie n'était en effet pas prioritaire par rapport à l'obligation d'assainir les lieux pour les rendre accessibles aux publics.

Figure 5

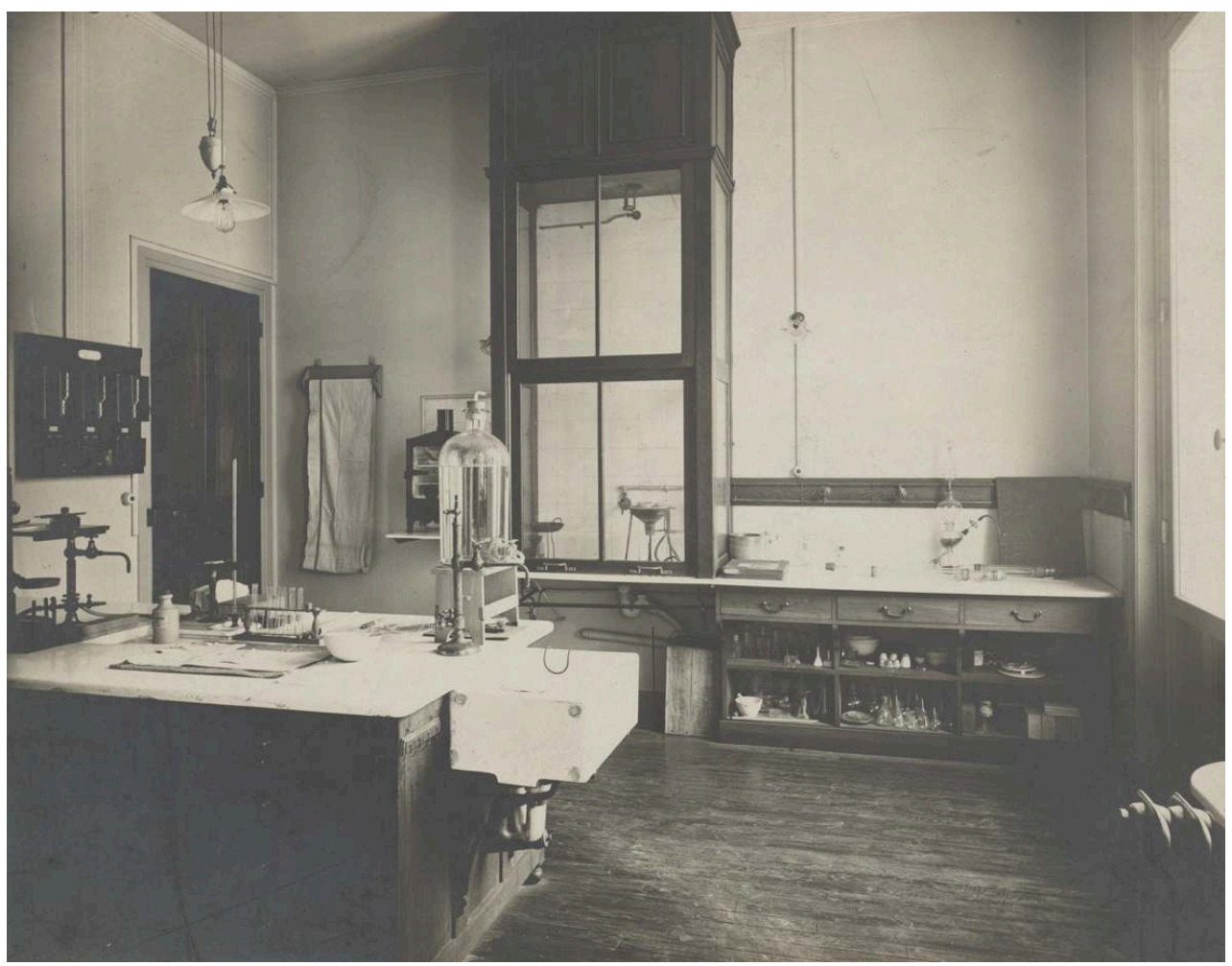

Laboratoire de chimie de Marie Curie en 1922.

(c) Musée Curie/coll. ACJC. 


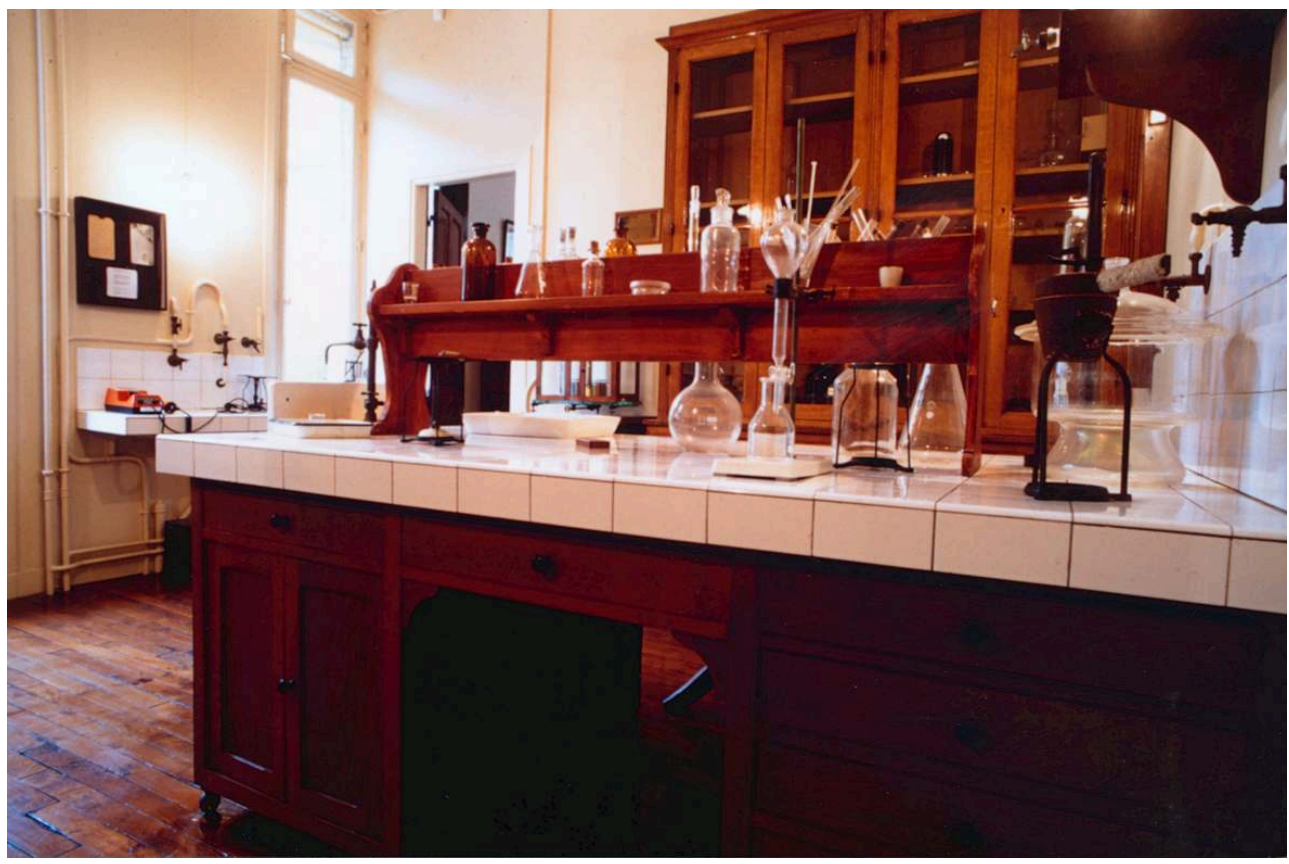

Laboratoire de chimie de Marie Curie après sa restauration.

Phot. Paty, Rachel, 1998. @ Rachel Paty /Musée Curie/coll. Institut du radium.

En 1989, le CNRS détache une deuxième personne pour contribuer au classement et à la communication des archives et recevoir davantage de groupes de visiteurs, sur rendezvous. Le public se diversifie et se compose à la fois de scientifiques, de chercheurs, de personnalités de passage, d'étudiants mais également, du « grand public » qui bénéficie de la possibilité de visiter les lieux à partir du début des années 1990. Pour répondre aux demandes de visite, de plus en plus nombreuses, l'AJC, puis l'Institut Curie proposent des vacations à des étudiants pour accueillir les visiteurs. À partir de 1992, le «musée Curie » est ouvert au public tous les après-midis de la semaine.

\section{La création d'une unité mixte}

La popularité croissante de Marie et Pierre Curie s'accompagne d'une modification des statuts de l'AJC, pour élargir et adapter ses missions: les Joliot-Curie sombrent progressivement dans l'anonymat, et c'est la figure de Marie Curie qui domine désormais dans l'esprit du public. L'AJC, qui devient l'Association Curie et Joliot-Curie (ACJC), dont les nouveaux statuts sont déposés le 2 mars 1993, vise à « faire connaître la vie et l'œuvre de Pierre et de Marie Curie, de Frédéric et d'Irène Joliot-Curie, afin d'en perpétuer la mémoire; de contribuer à la préservation et à la mise en valeur de leurs écrits, de leurs travaux et de tout document ou objet les concernant». Cette modification se traduit par une volonté d'amener le public vers une compréhension de la place de la science dans le monde actuel.

En parallèle, l'ACJC effectue des démarches auprès du CNRS et de l'Institut Curie pour proposer la création d'une structure pérenne. C'est le 17 juin 1994 que le musée et les archives Curie reçoivent leur statut actuel d'Unité mixte de service (UMS), sous l'intitulé « musée et archives de l'Institut du radium : Pierre et Marie Curie, Irène et 
Frédéric Joliot-Curie ». Cet acte officiel entre le CNRS et l'Institut Curie marque la naissance de l'actuel musée Curie, qui se distingue progressivement de l'ACJC, dont la présence se fera de plus en plus lointaine.

Le 20 avril 1995, Pierre et Marie Curie entrent au Panthéon, selon le souhait du président de la République François Mitterrand. La même année, un billet de 500 francs à leur effigie est créé par la Banque de France. L'affluence des visiteurs lors des visites organisées à l'Institut Curie en 1995, la célébration du $75^{\mathrm{e}}$ anniversaire de la création de la fondation Curie et celle du centenaire de la découverte de la radioactivité en 1996, conduisent à l'agrandissement et à l'organisation d'un réel espace d'exposition et à la rénovation de l'amphithéâtre, rendant les lieux plus fonctionnels pour présenter les collections et accueillir les visiteurs (fig. 7).

Figure 7

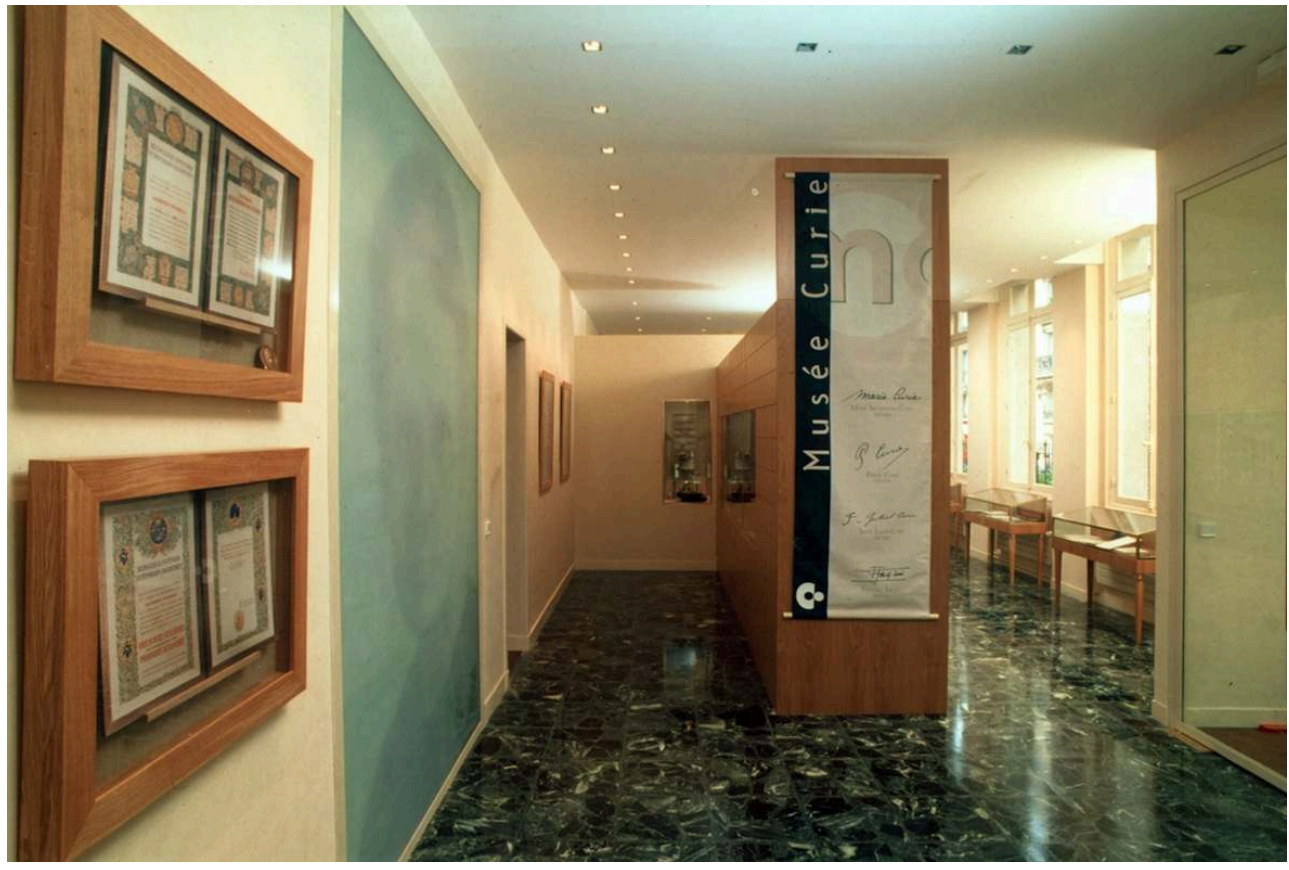

Vue du musée Curie après sa rénovation en 1996.

Phot. Gruner, C. ( ) Musée Curie/coll. ACJC.

En 1996, les treize universités de Paris, héritières de la faculté des sciences, font une donation à l'Institut Curie ${ }^{1}$ de l'ensemble immobilier de l'ancien Institut du radium, qui change ainsi de propriétaire. C'est cet Institut de recherche et de soins contre le cancer qui va financer une grande partie des travaux de rénovation.

Les archives quittent leur bâtiment d'origine, où se situent les salles historiques et la nouvelle exposition permanente, pour aller rejoindre un petit local de l'hôpital, rue Louis-Thuillier: une séparation physique et fonctionnelle est ainsi opérée entre les lieux de visite (musée) et de consultation des archives (recherche).

Une campagne de sensibilisation à la conservation des instruments scientifiques anciens et devenus obsolètes, qui ornent les étagères de certains bureaux ou laboratoires, est organisée au sein de l'Institut Curie. Cette démarche conduit à collecter quelques appareils, qui rejoignent ainsi les collections historiques du laboratoire Curie, et renforce le positionnement de l'UMS comme lieu de conservation 
du patrimoine scientifique. Les instruments demeurent cependant peu étudiés et délaissés, notamment en raison de leur accès, rendu difficile, voire impossible, compte tenu des conditions de stockage, dans un local exigu et inadapté dans les sous-sols, et de la radioactivité résiduelle de certains appareils. Un inventaire et des prises de vue des instruments du laboratoire sont cependant réalisés afin de faciliter la gestion de cette collection, notamment pour des prêts dans le cadre d'expositions temporaires ou itinérantes.

En 1997, un troisième poste est affecté par le CNRS à l'unité pour prendre en charge la gestion des fonds photographiques déposés par la famille à l'ACJC. Avec plusieurs étudiants vacataires recrutés pour accueillir les visiteurs et animer des conférences les après-midi, des doctorants en histoire des sciences et le personnel permanent, soit près d'une dizaine de personnes au total, le musée Curie connaît alors un succès grandissant auprès du public et l'équipe se structure. Par ses réalisations, le musée Curie confirme son intégration dans la communauté des professionnels du patrimoine et des musées. En 1999, à la suite de l'exposition "Les rayons de la vie», organisée au Muséum national d'histoire naturelle, les collections s'enrichissent de quelques appareils médicaux et d'une centaine d'ouvrages sur la cancérologie. Un réaménagement de l'espace d'exposition permanente du musée Curie est alors opéré pour améliorer la présentation des collections et accorder une partie à l'histoire des premières utilisations médicales des rayonnements.

\section{Le tournant des années 2000}

En 2003, les archives, les documents et le personnel déménagent dans des locaux mieux adaptés à la conservation et à la consultation des ressources historiques, au 70 rue Mouffetard. L'accueil des lecteurs est ainsi facilité, mais ce déplacement de quelques centaines de mètres amplifie encore la séparation entre d'une part, les lieux historiques accessibles au public, le musée, et d'autre part, les archives et le personnel de l'unité.

À partir de 2003, année de la célébration du centenaire du prix Nobel de physique attribué au couple Curie, le musée s'intègre à de nombreux événements (journées Portes ouvertes de l'Institut Curie, Fête de la science, Journées du patrimoine...) et propose des activités scientifiques et culturelles de plus en plus diversifiées: conférences et visites, expositions itinérantes ou temporaires, parcours organisés dans des autobus anciens "sur les traces de Pierre et Marie Curie », ateliers et animations pédagogiques, etc. Ces actions contribuent à une diversification et à un élargissement des publics.

Grâce à ces événements et à des ouvertures ponctuelles les week-ends, la fréquentation du musée augmente sensiblement, tandis que les demandes spécialisées sont également de plus en plus nombreuses pour des recherches et des projets éditoriaux ou culturels. En 2005, à l'occasion de l'Année internationale de la Physique, des instruments sont restaurés et remis en fonctionnement pour reproduire des mesures de radioactivité telles qu'elles se pratiquaient au temps des Curie et un conte pour les enfants sur Marie Curie est créé 2 . En 2006, une exposition sur Pierre Curie est produite au Panthéon, en collaboration avec l'ESPCI, et une journée de célébration du premier cours de Marie Curie à la Sorbonne est organisée à l'UPMC. Une exposition sur Frédéric Joliot-Curie est également montée en 2008 , et présentée à la mairie du $\mathrm{V}^{\mathrm{e}}$ arrondissement puis au siège du CNRS. 
Le musée Curie, de taille modeste (une centaine de mètres carrés) et ne disposant pas d'espaces pour des expositions temporaires, doit ainsi réaliser de nombreux projets " hors les murs ", afin de faire connaître son patrimoine scientifique.

Une dizaine d'années après la création de l'UMS, les activités et réalisations du musée montrent une réelle évolution. Le rayonnement international du musée Curie et la notoriété de la «famille aux cinq prix Nobel» suscitent l'intérêt grandissant de l'Institut Curie, qui célèbre en 2009 le centenaire de sa création et qui prend désormais en charge la majeure partie du fonctionnement de l'unité et le salaire de 4 postes permanents sur 5. L'équipe ainsi renforcée se spécialise dans la médiation scientifique et culturelle et continue de développer son expertise en histoire des sciences. À l'inverse, le CNRS se désinvestit progressivement de l'unité et ne finance plus qu'un seul poste permanent.

2 En 2007, la famille fait don à la BnF des archives Joliot-Curie, avec une convention de dépôt à l'Institut Curie: ces fonds changent ainsi de propriétaire, mais pas de localisation, ce qui permet au musée Curie de proposer aux lecteurs un ensemble thématique autour de l'histoire scientifique et sociale de la radioactivité. Les fonds d'archives concernant les Curie et les Joliot-Curie sont ainsi partagés entre deux lieux principaux, la BnF et le musée Curie.

Suite au décès, fin 2007, d'Ève Curie-Labouisse, fille cadette de Pierre et Marie Curie, un legs d'un million de dollars est affecté au musée. Membre du conseil d'administration de l'Institut Curie jusqu'en 1967, elle réclamait davantage d'égards et de mètres carrés à la mémoire de ses illustres parents ${ }^{3}$ et son legs a permis d'initier un nouveau projet de rénovation. Cependant, une très faible radioactivité résiduelle avait été détectée au niveau du sol de certaines pièces: les travaux de rénovation n'ont pu se dérouler qu'une fois cet assainissement effectué, qui a entraîné un retard de plusieurs mois dans le calendrier prévisionnel. Ainsi, en 2011, décrétée Année internationale de la Chimie et centième anniversaire du prix Nobel de chimie de Marie Curie, le musée Curie a été fermé au public. Les multiples sollicitations provenant du monde entier ont cependant été honorées, et de nouveaux projets ont été réalisés en parallèle de la progression du programme muséographique. Des expositions itinérantes, un conte pour enfants, un film documentaire et de nombreuses conférences et projets ont été produits en 2011, grâce à l'implication de l'ensemble des collaborateurs du musée, apportant une meilleure visibilité internationale du musée Curie.

Peu avant la fin des travaux, en mai 2012, la visite de François Hollande (fig. 8), rendant hommage à Marie Curie, fut un autre événement qui inscrivit encore davantage ce lieu de mémoire comme un élément du paysage culturel français. En déposant une gerbe à la mémoire de Marie Curie dans le petit jardin de son ancien Institut du radium, le président de la République salua la savante, qui symbolise l'internationalisation de la science, la réussite féminine et la recherche pure et désintéressée, en présence des petits-enfants de Pierre et Marie Curie. 
Figure 8

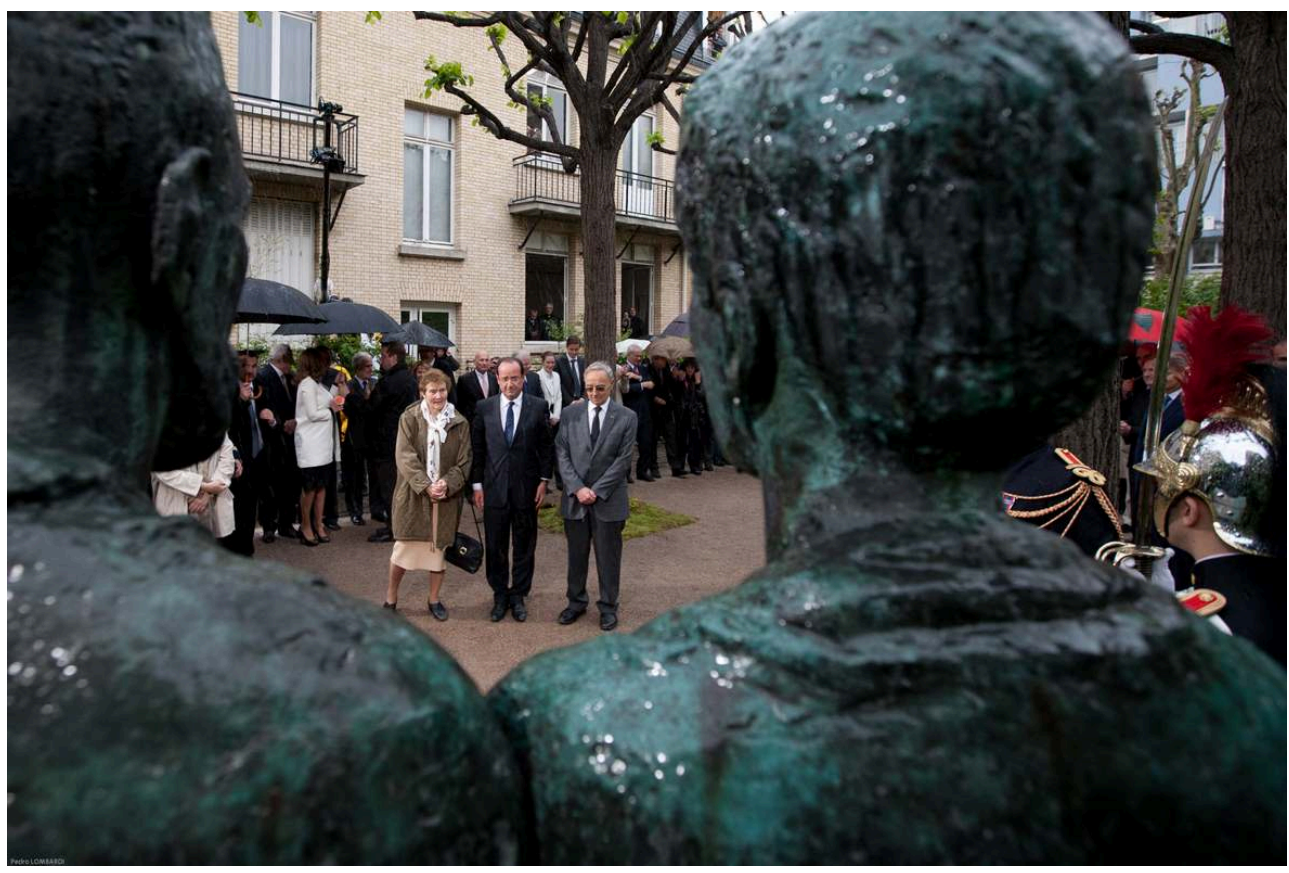

Le président François Hollande entouré des petits enfants des Curie, Hélène Langevin-Joliot et Pierre Joliot lors de la cérémonie en hommage à Marie Curie le jour de sa prise de fonctions, le 15 mai 2012. Phot. Lombardi, Pedro, 2012. (c) Pedro Lombardi /Institut Curie.

Le musée rouvre ses portes à la veille des Journées européennes du patrimoine, en septembre 2012 et le public découvre un nouveau parcours d'exposition, dans un espace redessiné par l'architecte Philippe de Potestad (fig. 9). Les instruments scientifiques y retrouvent une place de choix tout au long de la visite, et le parcours se trouve enrichi d'originaux et de reproductions de documents, présentés dans des vitrines, sur des panneaux ou à travers des écrans: l'apport du numérique pour la présentation des œuvres est indiscutable, tant du point de vue de la conservation préventive et de l'étude des collections (on ne manipule plus les originaux, mais leur copie numérique) que de celui de la médiation scientifique. 
Figure 9

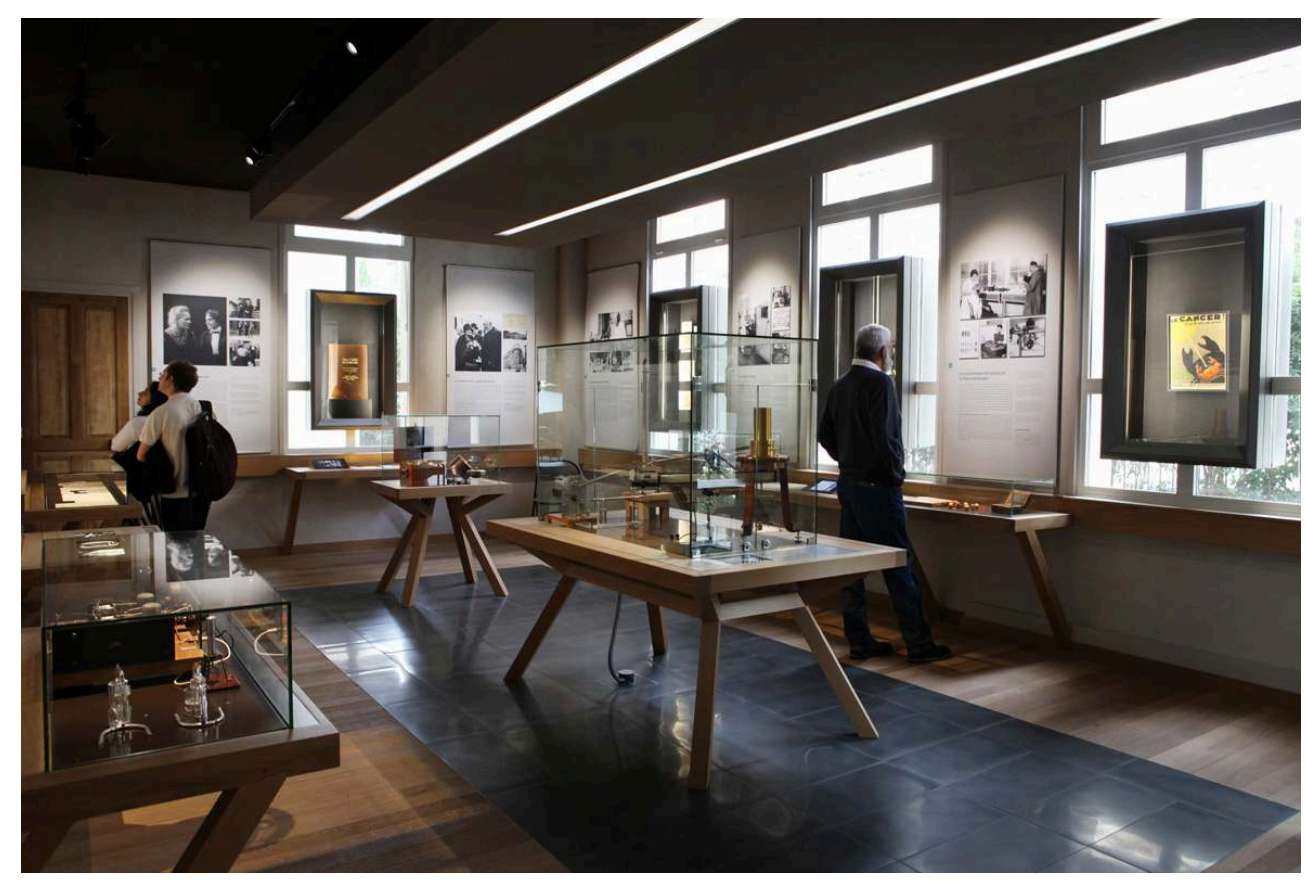

Salle principale d'exposition permanente du Musée Curie.

Phot. Hargoues, Christophe, 2012. (c) Christophe Hargoues/Musée Curie.

Certains appareils scientifiques ont connu les étapes successives de transformation des lieux, parmi lesquels les instruments qui composent la « méthode Curie » pour mesurer la radioactivité. Utilisée pour effectuer des mesures précises des rayonnements dès la fin du XIX siècle, cette méthode quantitative repose sur l'usage de trois instruments principaux: un électromètre à quadrants, une chambre d'ionisation et un quartz piézoélectrique (fig. 10, 11, 12). Fabriqués en série, ces appareils équipaient plusieurs salles du laboratoire dès le début des activités de l'Institut du radium et de nombreux spécimens ont été conservés. Ainsi, grâce à un travail de restauration et de remise en fonctionnement réalisé par un spécialiste des instruments anciens, ces appareils reprennent vie lors de démonstrations pour le public, une fois par mois. La médiation autour de ces appareils permet notamment d'illustrer les travaux historiques de Pierre et Marie Curie, mais aussi de détailler leur démarche scientifique et de sensibiliser le public à l'expérimentation, à la radioprotection et à la métrologie. 
Figure 10

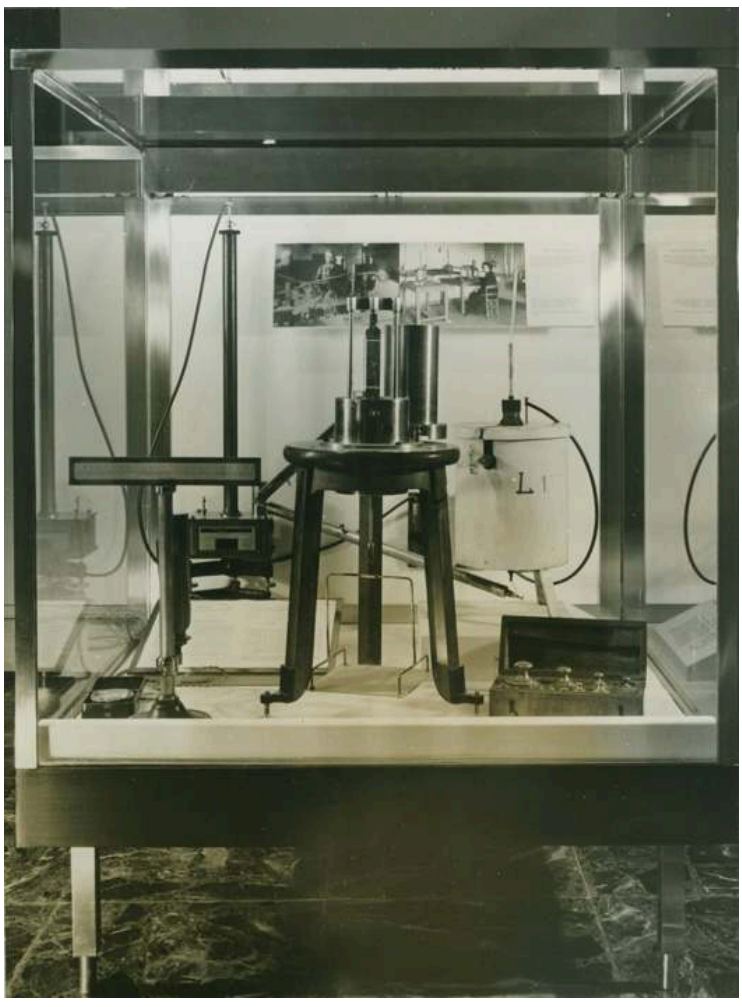

Vitrine « mesure de la radioactivité » dans l'espace d'exposition du pavillon Curie en 1964. (c) Musée Curie/coll. Institut du radium. 
Figure 11

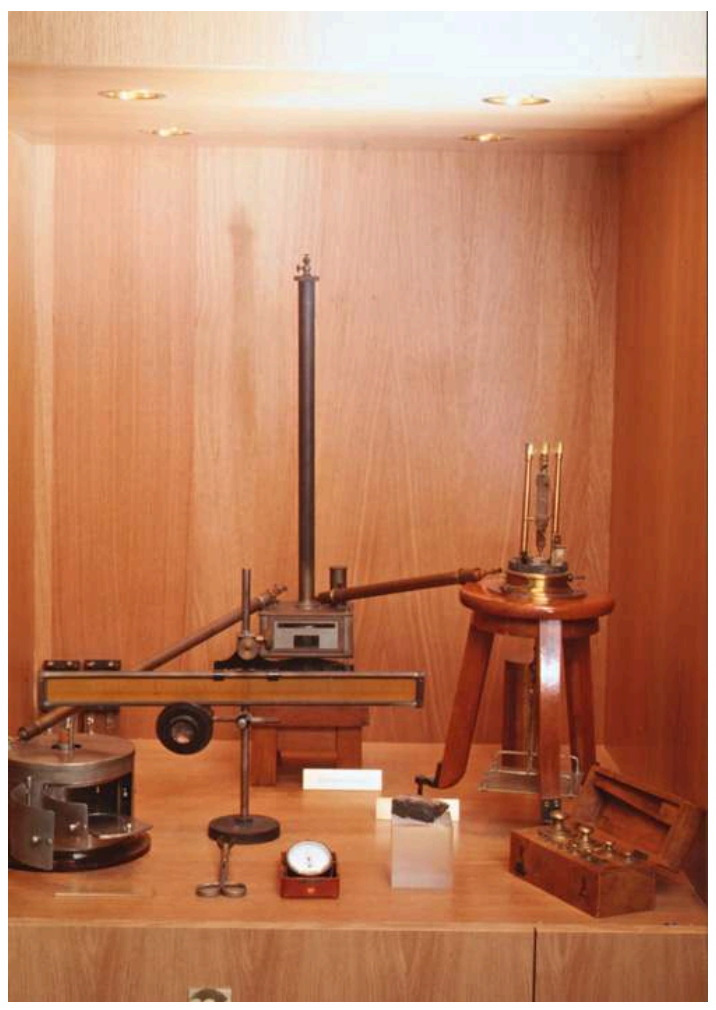

Vitrine «mesure de la radioactivité » dans le musée Curie après sa rénovation en 1996. Phot. Gruner, C., 1996. (C) C. Gruner /Musée Curie/coll. ACJC. 
Figure 12

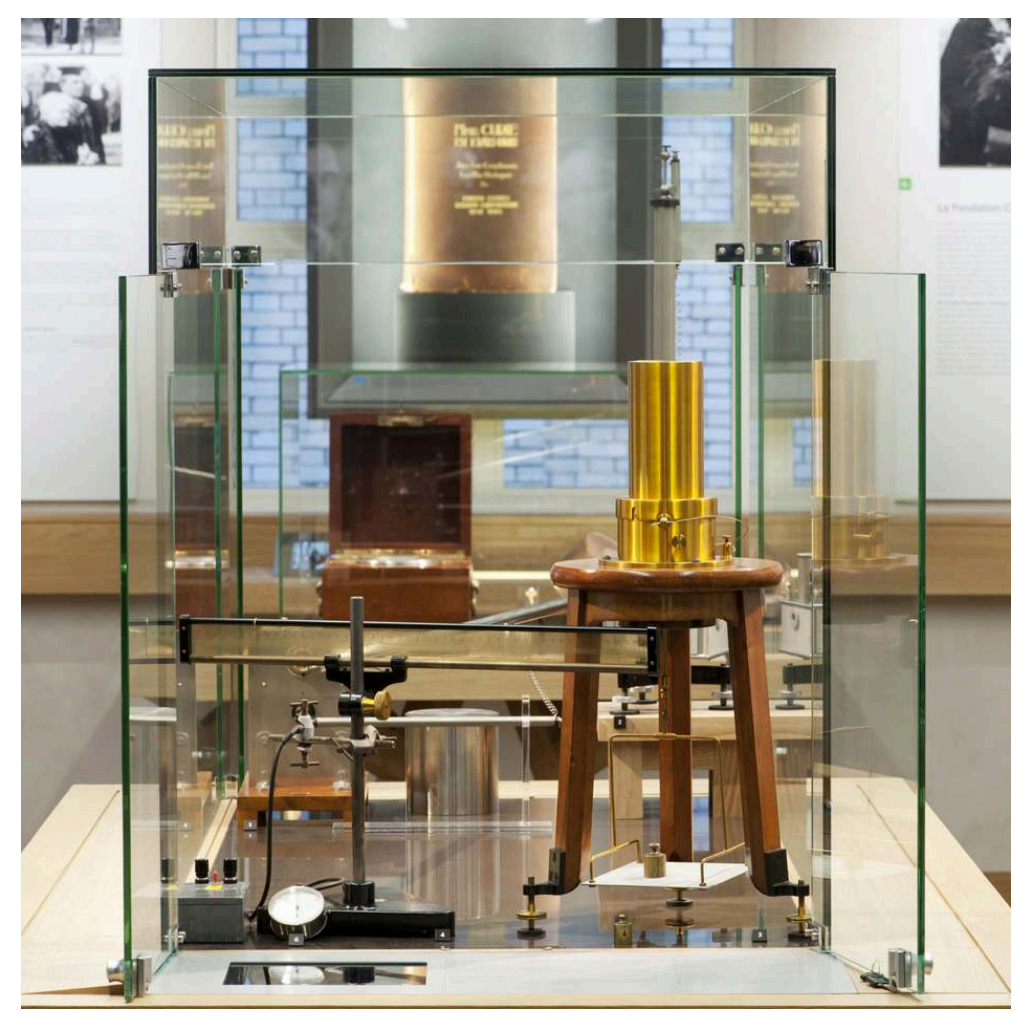

Vitrine « la méthode curie » du musée Curie en 2012. Les appareils restaurés peuvent être mis en fonctionnement pour des démonstrations de mesures de radioactivité.

Phot. Lenormand, Sacha, 2012. @ Sacha Lenormand/Musée Curie.

$\mathrm{Au}$ centre du parcours, qui propose quatre thématiques (la famille aux cinq prix Nobel, le radium entre mythe et réalité, le laboratoire Curie, la fondation Curie et la lutte contre le cancer), se trouvent les pièces historiques, le bureau de Marie Curie et son petit laboratoire de chimie reconstitué. Maintenir l'équilibre et le dialogue entre une certaine modernité et les lieux historiques est au centre du projet architectural, dont la réussite repose sur le fait que l'espace agrandi offre un meilleur confort de visite et un contenu plus riche et évolutif4.

Contrairement à ce que l'on pourrait penser au premier abord, cette rénovation n'est pas un aboutissement en soi, mais plutôt un moyen de créer une nouvelle dynamique pour valoriser ce patrimoine scientifique. C'est donc un nouvel élan pour le musée Curie, qui dispose désormais d'un espace de médiation mieux adapté aux publics et d'un accès vers le jardin, qui abrite désormais des expositions temporaires de plein air (fig. 13). 


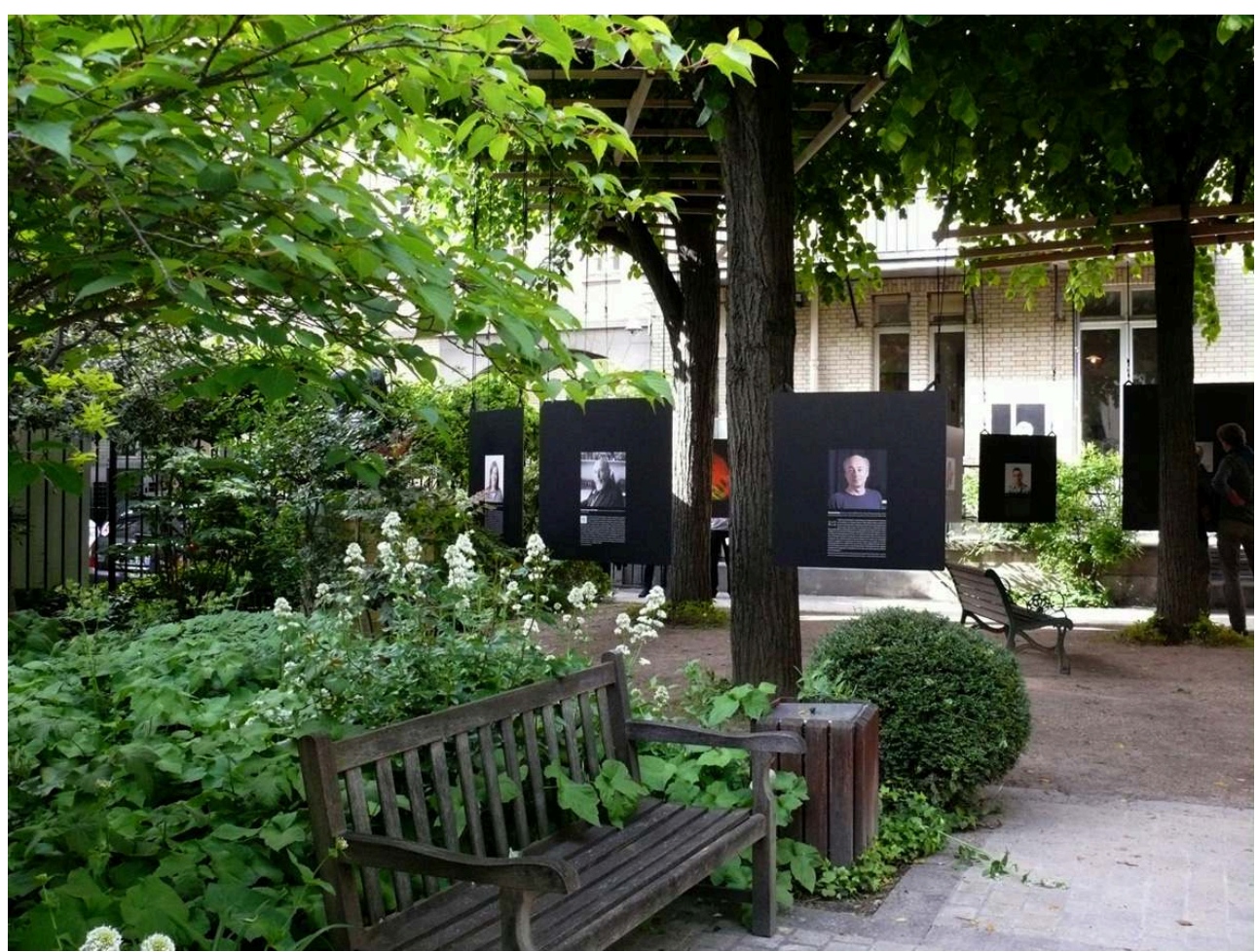

Exposition «Portrait(s) de cellules » dans le jardin du musée Curie, 2013.

(c) DR/Musée Curie.

\section{État des lieux}

Comme nous l'avons vu plus haut, différents événements ont donc progressivement conduit à la constitution de collections et à la conservation d'un ensemble formé non seulement de lieux historiques, mais aussi d'un riche patrimoine scientifique (instruments, archives et documents).

40 Les collections du musée Curie regroupent ainsi près d'un millier d'objets et instruments scientifiques, conservés dans un petit local exigu et non ventilé, dans le sous-sol du bâtiment du musée. Plusieurs fuites d'eau sont à déplorer ces dernières années et ce matériel doit être transféré dans un local plus vaste et mieux adapté à la gestion de ces objets historiques. Contrairement aux archives et autres documents scientifiques, les appareils du laboratoire n'ont été que peu étudiés depuis l'origine du musée Curie. Les enjeux des prochaines années reposent sur la possibilité ou non de décontaminer les pièces d'instruments qui portent encore les traces radioactives de leur usage au laboratoire. Ce sujet est complexe et plusieurs études sont en cours pour tenter de préserver pour les générations futures un patrimoine historique, malgré sa contamination par le radium.

41 Par ailleurs, plusieurs dizaines de milliers de documents sont conservés, inventoriés et consultés au centre de ressources historiques du musée : des archives manuscrites ou tapuscrites, des articles de presse, des fonds iconographiques et audiovisuels ${ }^{5}$, des ouvrages et des revues anciennes. La qualité et la diversité typologique de ces fonds en font une référence internationale dans son domaine de compétence: l'histoire des 
familles Curie et Joliot, de leurs laboratoires, de leurs découvertes et de tout ce qui s'y rattache, mais aussi celle du $D^{r}$ Claudius Regaud et du laboratoire Pasteur, de la radiothérapie et de la fondation Curie. Ainsi, de nombreux lecteurs (historiens, chercheurs, collectionneurs, journalistes, étudiants, lycéens...) sont accueillis afin de profiter de cette richesse patrimoniale. De nombreuses demandes spécialisées sont également traitées par courriel.

Pour des raisons sanitaires, le centre de ressources historique de la rue Mouffetard a été fermé en 2013 et il est actuellement installé dans un lieu provisoire, en attendant une localisation pérenne, à proximité immédiate du musée, dans les anciens bâtiments de l'Institut du radium. Le plan de développement du musée Curie dans les prochaines années prévoit de rassembler définitivement l'ensemble de ses collections (archives et objets) dans un petit pavillon de l'Institut du radium. Regrouper la «mémoire des lieux ", portée par les ressources historiques et les objets matériels, dans les « lieux de mémoire » de l'Institut du radium, est ainsi un moyen de renforcer et de faire rayonner cet ensemble patrimonial.

Malgré sa répartition sur plusieurs sites, musée et centre de ressources historiques, ce patrimoine est devenu au fil des décennies une référence sur l'histoire de la famille aux cinq prix Nobel, de la radioactivité et de la radiothérapie. De nombreux événements et commémorations vont ainsi progressivement inscrire le musée Curie comme un acteur important pour le rayonnement international de la science française.

\section{Les trois actes de la patrimonialisation}

La visite du président de la République en 2012, puis la labellisation «maisons des Illustres " par le ministère de la Culture et de la Communication, du lieu où Marie Curie, puis Irène et Frédéric Joliot-Curie ont effectué une partie de leurs travaux, octroient au patrimoine scientifique du musée Curie une dimension culturelle. Les figures célèbres sont désormais des "êtres culturels » et les lieux historiques deviennent patrimoniaux.

Dans son ouvrage La fabrique des Illustres, Saurier ${ }^{6}$ propose une analyse heuristique qui permet de résumer en trois actes principaux et en trois périodes le processus de « patrimonialisation » du musée Curie.

46 Le premier acte consiste en la sauvegarde des lieux historiques à la mémoire des personnes admirées. Pour le musée Curie, l'acte fondateur est donc la préservation des pièces historiques (bureau et laboratoire) à la fin des années 1950 et du matériel scientifique du laboratoire (archives, instruments, objets), progressivement inventorié dans les décennies suivantes, à la mémoire des Joliot-Curie et des Curie. Jusqu'aux années 1990, les nombreuses actions de l'AJC marquent une première étape dans la diffusion des connaissances sur la «famille aux 5 prix Nobel», qui s'inscrit dans la mémoire collective. Cette première période est également importante dans la constitution d'un patrimoine matériel: plusieurs dons sont ainsi venus enrichir le fonds initial du laboratoire Curie.

Le second acte se déroule au milieu des années 1990, période pendant laquelle nous constatons une transformation des lieux historiques en lieux de mémoire et des personnes admirées en figures admirables. La notoriété grandissante dans le monde de Marie Curie et les nombreuses publications sur les travaux du couple Curie ont conduit à un 
déplacement : la mémoire des savants admirés change d'échelle et passe du cercle des initiés (famille, anciens collaborateurs, historiens, scientifiques...) à celui d'un public plus large.

Ce sont les années 2000 qui constituent le $3^{\mathrm{e}}$ acte de "patrimonialisation " du musée Curie, période pendant laquelle les figures admirables deviennent figures célèbres tandis que les lieux de mémoire se transforment en lieux patrimoniaux.

Cette part mémorielle et culturelle des "figures célèbres" est primordiale pour le musée Curie car elle lui procure une forme de protection. Marie et Pierre Curie, leurs découvertes, font partie du patrimoine culturel national, et il ne viendrait à l'esprit de personne aujourd'hui de remettre en cause l'intérêt historique des pièces qui ont abrité la célèbre savante, ce qui n'aurait pas forcément été le cas de son vivant, malgré ses deux prix Nobel' ${ }^{7}$. Ainsi, malgré l'absence de protection juridique au sens strict du terme, les trois bâtiments qui constituent l'ancien Institut du radium, le pavillon Curie (dans lequel se trouve le musée), le pavillon Pasteur et le petit pavillon, ont été préservés, au sein d'un campus scientifique dont le patrimoine architectural est le reflet de l'essor des institutions scientifiques dans les trois premières décennies $d u \mathrm{xx}^{\mathrm{e}}$ siècle. Cependant, la tension entre patrimoine et modernité perdure, malgré l'intérêt grandissant des scientifiques pour leur patrimoine. En effet, la communauté scientifique privilégie l'efficacité, la production scientifique, l'avenir et l'innovation, au détriment parfois de la préservation de la mémoire du passé.

50 Aussi, il est fréquent de jeter les "vieux papiers " ou le matériel ancien, sauf s'il présente un intérêt décoratif ou ornemental quelconque. De même, les installations techniques, nécessaires au fonctionnement des laboratoires, sont parfois fixées directement sur la façade de certains bâtiments historiques, privilégiant l'efficacité à l'esthétique ou à la protection du patrimoine. Cet état de fait ne doit cependant pas freiner le cheminement vers une protection plus pérenne de cet ensemble immobilier de la Montagne Sainte-Geneviève, sans toutefois interférer avec le bon déroulement des recherches scientifiques qui s'y déroulent. La cohabitation d'une structure patrimoniale et culturelle au sein d'un environnement scientifique en activité est un exercice complexe, qui repose sur le principe du respect mutuel et de la communication. L'évocation in situ de l'histoire des sciences, à travers des personnages et des événements, est une forme de médiation qui pourrait bénéficier à la fois au tourisme culturel et au rayonnement scientifique de ces institutions, pour la plupart centenaires et riches d'une histoire prestigieuse.

51 Le patrimoine mobilier et immobilier du musée Curie concerne ainsi à la fois des lieux, des objets et des personnes. À la fois musée et lieu patrimonial, cette institution remplit plusieurs fonctions auprès des publics : c'est un "lieu de mémoire authentique », un espace mémoriel dépositaire d'une identité, celle d'une famille, d'une communauté ; c'est aussi un lieu « cultuel » où le visiteur se rend sur les traces du savant-modèle de Marie Curie; c'est un lieu «culturel» dans le sens où il présente au public des collections patrimoniales; enfin, c'est un lieu de médiation et de communication scientifiques ${ }^{8}$.

De taille relativement modeste, le musée Curie est polymorphe et possède de nombreux atouts qui lui ont permis d'affirmer son existence dans un environnement scientifique dans lequel le patrimoine est rarement une priorité. 


\section{NOTES}

1. - L'Institut Curie, fondation privée reconnue d'utilité publique, est issu de la fusion de l'Institut du radium et de la fondation Curie dans les années 1970.

2. - Ces opérations ont été réalisées grâce à une subvention de la DDRT-DRAC île-de-France.

3. - Ève Curie aurait même proposé au CA que l'ensemble du pavillon Curie, haut de 2 étages avec un sous-sol et comprenant un amphithéâtre, soit consacré en totalité aux activités du musée Curie. Difficilement compatible avec le développement des recherches en cancérologie et aux besoins croissants des laboratoires, cette demande n'a pu être satisfaite.

4. - Depuis peu, une application évolutive Google est disponible pour découvrir les collections autrement, à partir d'une tablette ou d'un smartphone (g.co/museecurieapp).

5. - Voir les sites : http://www.numerique.culture.fr/pub-fr/document.html?id=FR-DCX0238_2576a086; http://www.calames.abes.fr/pub/curie.aspx\#details?id=FileId-450 ; http://www.calames.abes.fr/pub/curie.aspx\#details?id=FileId-1069 [consultés le 19/05/2016].

6. - GUZOWSKI-SAURIER, Delphine. La fabrique des illustres: Proust, Curie, Joliot et lieux de mémoire. Paris : Non Standard, 2013.

7. - De fait, plusieurs lieux historiques n'ont pas résisté au développement des laboratoires et institutions scientifiques au xxe siècle : le « hangar de la découverte » du polonium et du radium n'a pas été conservé à l'ESPCI, tout comme l'amphithéâtre de l'annexe de la faculté des sciences au 12 rue Cuvier, où Pierre Curie enseignait. Seuls les murs d'un petit bâtiment du "labo Curie » dans la cour de ce qui est devenu l'Institut de physique du globe de Paris ont été conservés, malgré leur faible intérêt architectural, pour y abriter une salle de réunion dont la décoration propose une évocation des travaux des Curie.

8. - Voir : http://ceroart.revues.org/4160 ; et les 6 photographies du fonds Kertesz conservé à la médiathèque de l'architecture et du patrimoine et accessibles sur la base mémoire, mai 1932 : notices AP72L003469 et AP72L003470 [consultés le 19/05/2016].

\section{RÉSUMÉS}

Le musée Curie, situé dans l'ancien laboratoire Curie de l'Institut du radium, est devenu au fil des décennies un musée d'histoire des sciences, qui préserve et valorise des collections scientifiques au sein de l'actuel Institut Curie. Notre article propose une rétrospective de la manière dont cet ensemble patrimonial s'est constitué et continue de se construire, et propose un état des lieux de la situation actuelle.

Over the years, the Curie Museum, located in the former Curie Laboratory of the Radium Institute, has become a museum of the history of science. Its scientific collections are preserved within the current Institut Curie, and are located at two different sites. Our article offers a retrospective account of how this heritage ensemble was formed and gives an overview of the current situation. 
INDEX

Mots-clés : patrimoine scientifique, laboratoire, radioactivité, musée, histoire des sciences, Institut du radium, Curie, Joliot-Curie, patrimonialisation

Keywords : Musée Curie, Institut Cure, Pierre Curie, Marie Curie, Frédéric Joliot-Curie, Irène Joliot-Curie, radioactivity, history of science, scientific collections

\section{AUTEURS}

RENAUD HUYNH

Directeur du musée Curie, Institut Curie renaud.huynh@curie.fr

ADRIEN KLAPISZ

Responsable des collections, musée Curie, Institut Curie adrien.klapisz@curie.fr 